\title{
Robust Consensus of Multi-agent Systems with Bounded Disturbances
}

\author{
Lin Wang ${ }^{1}$ and Zhixin Liu $^{2}$ \\ ${ }^{1}$ Shanghai Jiao Tong University \\ ${ }^{2}$ Academy of Mathematics and Systems Science, Chinese Academy of Sciences
}

China

\section{Introduction}

Recent years have witnessed an increasing interest in the coordination of distributed multi-agent systems. In this area, one of the most fundamental problems is the consensus problem, which has broad connections with a wide range of disciplines including statistical decision theory [1, 2], distributed computing [3, 4], biology [5, 6], and cooperation of multi-agent systems[7, 8].

Consensus roughly speaking is characterized as a collection of agents with locally sensed information or limited inter-component communications seeking to reach a common value. A basic consensus protocol in the context of multi-agent systems usually consists of a information exchange network, in which each agent updates its state by forming a convex combination of the states of its neighbors and itself. Some sufficient consensus conditions for the heading angles of a group of agents modeled by Vicsek et al. [5] are presented in $[9,10]$. Some less restrictive conditions were obtained in $[12,13]$, where consensus is ensured if there exists a spanning tree in the union of the information exchange networks. Many other generalizations have been devoted to different types of agent dynamics and different topologies of information exchange networks, such as nonlinear consensus protocol [14], consensus of agents modeled by double integrators [15], consensus algorithm with cohesion, separation and alignment rules [16, 17], consensus over random networks [18, 19], consensus of networked agents with time-delays [20,21].

Most of the previously mentioned references used noise-free state iteration, that is they assume the information exchange between agents is accurate. This assumption would obviously be inappropriate in real distributed systems, since there are various kinds of noises during the sending, transmission and receiving of information. Consensus of distributed systems with noise disturbance is an important challenge, and now there are only a few results. The average-consensus control with fixed topology and additive input noises is investigated in [22], where the long term consensus error is minimized by a least mean square optimization method. [23] considered the consensus protocol with fixed topology and independent identically distributed noises, and used stochastic Lyapunov functions to establish mean square consensus. The extension to the case of time-varying topologies is carried in [24], where some sufficient conditions are given for mean square average-consensus and almost sure consensus. [11] further investigated decentralized adaptive synchronization for a stochastic model with uncertainties. Roughly speaking, the consensus algorithms 
in these researches are essentially distributed stochastic approximation type consensus protocols, and the consensus gains are essential for the consensus properties.

In this paper, we investigate the consensus problem of multi agent systems with bounded disturbances. The information exchange topology is time-varying, and the consensus protocol has a general form

$$
x(t+1)=P(t) x(t)+w(t+1), t=1,2, \cdots,
$$

where $x(t) \in \mathbf{R}^{n}$ is the information state, $w(t) \in \mathbf{R}^{n}$ is the noise disturbance, and $\{P(t)\}$ is a sequence of stochastic matrices. Here, the noise is bounded, and does not require the zero-mean property needed by the previous stochastic approximation type consensus protocols. For the system, it is expected that small disturbance of $\{w(t)\}$ would give rise to small discrepancy of the states of agents from each other, namely robust consensus in this paper.

We first investigate the case that the information exchange between the agents is bidirectional. From the well-known relationship between the external stability and the internal stability, we know that the exponential stability of the corresponding homogeneous dynamical equation will play a crucial role. Based on this observation, we establish the equivalence among the robust consensus, the positivity of the second smallest eigenvalue of the weighted Laplacian matrix, and the connectivity of the union graph, where several basic results on stability of time-varying systems [28], product of nonnegative stochastic matrices[27, 31, 32], algebraic graph theory [30] and Markov chains [29] are useful.

Since there are a variety of practical applications where information only flows in one direction, the case of interaction topologies with directed information exchanges is also studied. In this case, we show that the robust consensus can be achieved under dynamically changing interaction topology if and only if the union of the collection of interaction graphs across some time intervals has a spanning tree frequently enough.

The paper is partially based on the results in $[25,26]$. Some new insights and simulations are added to illustrate the theoretical results. Section 2 recalls some basic notions and motivates the problem to be investigated; the case of undirected information exchange topology is studied in Section 3 while the directional case is studied in Section 4; Some simulation results are given in Section 5; and a brief conclusion is made in Section 6.

\section{Preliminaries and problem statement}

\subsection{Preliminaries}

The information exchange between agents can always be represented by directed/undirected graphs. A directed graph $G=(V, E)$ consists of a vertex set $V$ and an edge set $E=\{(i, j)\}$, where $V=\{1,2, \cdots, n\}$ is composed of the indices of all agents, and $E \subseteq V \times V$ is a set of ordered pairs of vertexes. As a comparison, an undirected graph means the pairs of vertexes in the edge set are unordered. If there is a directed edge from vertex $j$ to $i$, then $j$ is defined as a parent vertex and $i$ is a child vertex, which means that $i$ can receive the information from agent $j$. The neighbors of agent $i$ are denoted by $N_{i}=\{j \subseteq V:(j, i) \in E\}$. A path that connects $i$ and $j$ in a directed/ undirected graph $G$ is a sequence of distinct vertexes $i_{0}, i_{1}, i_{2}, \cdots i_{m}$, where $i_{0}=i, i_{m}=j$ and $\left(i_{l}, i_{l+1}\right) \in E, 0 \leq l \leq m-1$. A graph is called connected (or strongly connected for directed graph) if for every pair of distinct vertexes there is a path connecting them. A digraph is said to have a spanning tree if and only if there exist a vertex $i \in V$, called root, such that there is a path from $i$ to any other vertex. The union of a collection of graphs 
$\left\{G_{1}, G_{2}, \cdots, G_{h}\right\}$ with the same vertex set $V$, is a graph $G$ with vertex set $V$ and edge set equaling the union of the edge sets of those graphs.

The adjacency matrix $A=\left[a_{i j}\right]$ of a weighted graph $G$ is defined as $a_{i j}>0$ if $(j, i) \in E$. The adjacency matrix of a weighted undirected graph is defined analogously except that $a_{j i}=$ $a_{j i}, \forall i \neq j$, since the edge is unordered and the same for its two adjacent vertexes. The degree matrix $D=\left[d_{i j}\right]$ is a diagonal matrix with $d_{i i}=\sum_{j=1}^{n} a_{i j}$. The Laplacian matrix $L=\left[l_{i j}\right]$ is defined as $L=D-A$, which implies that 0 is one of its eigenvalues. Moreover, 0 is a simple eigenvalue if the graph is strongly connected [31]. For an undirected graph, $L$ is symmetric positive semi-definite. For a positive semi-definite matrix $B$, we arrange all its eigenvalues in a nondecreasing order: $0 \leq \lambda_{0}(B) \leq \lambda_{1}(B) \leq \cdots \leq \lambda_{n-1}(B)$. In some cases, we are interested in the second smallest eigenvalue $\lambda_{1}(B)$.

Some notations from nonnegative matrix theory are important for investigating the consensus property [29,31]. A matrix is nonnegative (positive) if all its entries are nonnegative (positive). Moreover, if the sum of each row satisfies $\sum_{j=1}^{n} a_{i j}=1, i=1, \cdots, n$, the matrix is called stochastic. A stochastic matrix $P$ is said to be indecomposable and aperiodic (SIA) if $\lim _{k \rightarrow \infty} P^{k}=\mathbf{1} v^{\tau}$, where $\mathbf{1}$ is a column vector of all ones and $v$ is some column vector. Define

$$
\lambda(P)=\min _{i, j} \sum_{s=1}^{n} \min \left(P_{i s}, P_{j s}\right) .
$$

If $\lambda(P)>0$, then $P$ is called scrambling matrix. For a matrix $P=\left[p_{i j}\right]_{n \times n}$, its associated directed graph $\Gamma(P)$ is a directed graph on $n$ nodes $1,2, \cdots, n$ such that there is a directed arc in $\Gamma(P)$ from $j$ to $i$ if and only if $p_{i j} \neq 0$ (cf. [31]).

\subsection{Problem statement}

Let $x_{i}(t) \in \mathbf{R}, i=1, \cdots, n$ represent the information state of agent $i$ at time $t$. As described in $[1,2,7-10]$, a discrete-time consensus protocol can be summarized as

$$
x_{i}(t+1)=\frac{1}{\sum_{j \in N_{i}(t)} a_{i j}(t)} \sum_{j \in N_{i}(t)} a_{i j}(t) x_{j}(t),
$$

where $a_{i j}(t) \geq 0$ represents the weighting factor, and $N_{i}(t)=\left\{j: a_{i j}(t)>0\right\}$ is a set of agents whose information is available to agent $i$ at time $t$.

In the real world, the outside interference and measurement error are unavoidable. Each agent receives in fact noisy information from its neighbors. Assume the resulting information of agent $j$ received by agent $i$ is the following form:

$$
y^{i j}(t)=x_{j}(t)+e^{i j}(t),
$$

where $e^{i j}(t)$ is the noise. The update law of agent $i$ under the influence of noise can be described as

$$
\begin{aligned}
x_{i}(t+1) & =\frac{1}{\sum_{j \in N_{i}(t)} a_{i j}(t)} \sum_{j \in N_{i}(t)} a_{i j}(t) y^{i j}(t) \\
& =\frac{1}{\sum_{j \in N_{i}(t)} a_{i j}(t)} \sum_{j \in N_{i}(t)} a_{i j}(t) x_{j}(t)+\frac{1}{\sum_{j \in N_{i}(t)} a_{i j}(t)} \sum_{j \in N_{i}(t)} a_{i j}(t) e^{i j}(t) .
\end{aligned}
$$


Let $G_{t}=\left(V, E_{t}\right)$ represent the neighbor graph that $(j, i) \in E_{t}$ iff $j \in N_{i}(t)$. Let $A(t)=\left[a_{i j}(t)\right]$ be the adjacency matrix that $a_{i j}(t)>0$ iff $j \in N_{i}(t)$. Let $D(t)$ be the associated degree matrix. Let $w_{i}(t)=\frac{1}{\sum_{j \in N_{i}(t)} a_{i j}(t)} \sum_{j \in N_{i}(t)} a_{i j}(t) e^{i j}(t)$. Then the matrix form of system (3) is

$$
x(t+1)=D^{-1}(t) A(t) x(t)+w(t), \quad t=1,2, \cdots,
$$

where $x(t)=\left[x_{1}(t), \cdots, x_{n}(t)\right]^{\tau}$ is the vector formed by the states of all agents, and $w(t)=$ $\left[w_{1}(t), \cdots, w_{n}(t)\right]^{\tau}$ is the noise vector. Define $P(t)=D^{-1}(t) A(t)$. It's easy to check that $P(t)$ is a stochastic matrix, and $P(t)=I-D^{-1}(t) L(t)$ with $L(t)$ being the Laplacian matrix. The system (4) can be rewritten as

$$
x(t+1)=P(t) x(t)+w(t), \quad t=1,2, \cdots .
$$

In this paper, we propose the following assumption on the matrix $A(t)=\left[a_{i j}(t)\right]$, which is simple and easily satisfied.

\section{Assumption $\Lambda$ :}

(1) For each $t, A(t)$ has positive diagonal entries, i.e. $a_{i i}(t)>0$;

(2) There exist two constants $\alpha, \beta>0$ such that $\alpha \leq a_{i j}(t) \leq \beta$ for all $a_{i j} \neq 0$.

Assumption $\Lambda(1)$ means that each agent can sense its own information, and Assumption $\Lambda(2)$ means that the information exchange between two neighboring agents has some bounds.

The purpose of this paper is to study the consensus property of system (4). Generally, by consensus we mean that for any two agents $i$ and $j$, their states satisfy $\lim _{t \rightarrow \infty}\left\|x_{i}(t)-x_{j}(t)\right\|=0$. In the presence of noise, we should not expect that the agents can reach consensus eventually. So, we introduce a concept- robust consensus to describe the influence of the noise to the behavior of the system. Define the distance between a vector $x$ and a subspace $X \subset \mathbf{R}^{n}$ as

$$
d(x, X)=\inf _{y \in X} d(x, y)=\inf _{y \in X}\|x-y\|,
$$

where $\|\cdot\|$ is the standard Euclidean norm. In this paper, we take $X$ as the space spanned by the vector $[1,1, \cdots, 1]^{\tau} \in \mathbf{R}^{n}$, i.e., $X=\operatorname{span}\left\{[1,1, \cdots, 1]^{\tau}\right\}$, and denote the orthogonal complement space of $X$ by $M$. Define a function set and a noise set as follows:

$$
\begin{aligned}
& K_{0}=\left\{f(\cdot) \mid f: R^{+} \rightarrow R^{+}, f(0)=0, f(\delta) \text { decreases to } 0 \text { as } \delta \rightarrow 0\right\} \\
& \mathcal{B}(\delta)=\left\{\{w(t)\} \mid \sup _{t \geq 0} d(w(t), X) \leq \delta\right\} .
\end{aligned}
$$

Definition 2.1. System (4) is said to be robust consensus with noise, if there exist a function $f(\cdot) \in K_{0}$ and a constant $T>0$ such that for any $\delta>0, x(0) \in \mathbf{R}^{n}$, and any sequence $\{w(t)\} \in \mathcal{B}(\delta)$,

$$
d(x(t), X) \leq f(\delta), t \geq T .
$$

Remark 2.1. If the noise vector $w(t)=c(t) \cdot \mathbf{1}$ with $c(t) \in \mathbf{R}$ being very large, then it may have strong influence on the states of the agents but have no influence on the consensus property, since the noise disturbance can be eliminated when considering the difference of the states between agents. This is the reason that we use $d(w(t), X)$ rather than $\|w(t)\|$ to describe the noise effect here. 


\section{Undirected information exchange topology}

In this section, we assume the information exchange topology is undirected, that is the weighted adjacency matrix $A(t)$ is symmetric. In this case, the Laplacian matrix $L(t)=$ $D(t)-A(t)$ is positive semi-definite.

The following theorem is our main result.

Theorem 1. Consider System (4) under Assumption $\Lambda$. If the weighted adjacency matrix $A(t)$ is symmetric, then the following three propositions are equivalent:

(i) The system (4) is robust consensus.

(ii) For the Laplacian matrix $L(t)$, there exists a constant $q>0$ such that

$$
\inf _{t \geq 0} \lambda_{1}\left(\sum_{k=t+1}^{k=t+q} L(k)\right) \neq 0 .
$$

(iii) There exists a constant $q>0$ such that for any $t \geq 0$, the union of the neighbor graphs $\left\{G_{t+1}, G_{t+2}, \cdots, G_{t+q}\right\}$ is connected.

For readability, we divide the proof into the following three subsections.

\subsection{The proof of (i) $\Rightarrow$ (ii)}

By introducing a suitable projection operator, we can translate the distance between a vector and the subspace $X$ into the norm of the projected vector, so the problem of robust consensus can be transformed into a certain robust stability in the subspace. We decompose the space $\mathbf{R}^{n}$ into two orthogonal subspaces $X$ and $M=X^{\perp}$. As $X$ and $M$ are closed subspaces, we know that for any $x \in \mathbf{R}^{n}$, there exists a unique pair of vectors $x_{0} \in M, x_{1} \in X$ such that $x=x_{0}+x_{1}$. Furthermore, according to the property of projection, we have $\left\|x_{0}\right\|=\left\|x-x_{1}\right\|=$ $\inf _{y \in X}\|x-y\|=d(x, X)$. Denote by $P_{M}$ the projector onto $M$. Then $P_{M} x=x$ if and only if $x \in M$, $P_{M} x=0$ if and only if $x \in X$, and

$$
\left\|P_{M} x\right\|=d(x, X) .
$$

Take a standard orthogonal base $e_{1}, e_{2}, \cdots, e_{n}$ in the space $\mathbf{R}^{n}$, where $e_{n}=\left[\frac{1}{\sqrt{n}}, \cdots, \frac{1}{\sqrt{n}}\right] \tau$. Then $X=\operatorname{span}\left\{e_{n}\right\}$ and $M=\operatorname{span}\left\{e_{1}, e_{2}, \cdots, e_{n-1}\right\}$.We can get a detailed form of the projector $P_{M}$ as follows:

$$
P_{M}=Q\left(\begin{array}{ccccc}
1 & 0 & \cdots & 0 & 0 \\
0 & 1 & \cdots & 0 & 0 \\
\cdots & \cdots & \cdots & \cdots & \cdots \\
0 & 0 & \cdots & 1 & 0 \\
0 & 0 & \cdots & 0 & 0
\end{array}\right) Q^{\tau}=I-\frac{1}{n} \mathbf{1} \cdot \mathbf{1}^{\tau}
$$

where $Q=\left[e_{1}, e_{2}, \cdots, e_{n}\right]$. The projector $P_{M}$ has the following property.

Lemma 1. If $P_{1}, P_{2}$ are stochastic matrices, and $P_{M}$ is the projector onto $M$, then we have

$$
P_{M} P_{1} P_{M} P_{2}=P_{M} P_{1} P_{2}
$$


Proof. By using the expression of the projector $P_{M}$ and the properties of the stochastic matrix, we have

$$
P_{M} P_{1} P_{M} P_{2}=P_{M} P_{1}\left(I-\frac{1}{n} \mathbf{1} \cdot \mathbf{1}^{\tau}\right) P_{2}=P_{M}\left(P_{1}-\frac{1}{n} \mathbf{1} \cdot \mathbf{1}^{\tau}\right) P_{2}=P_{M} P_{1} P_{2}
$$

Moreover, if $\left\{P_{i}, i=1, \cdots\right\}$ are stochastic matrices, by the above lemma and the fact that the product of stochastic matrices is still stochastic, we can deduce that

$$
\prod_{i=1}^{n} P_{M} P_{i}=P_{M} \prod_{i=1}^{n} P_{i}
$$

Now, letting the projector $P_{M}$ act on both sides of the system (5), we have

$$
P_{M} x(t+1)=P_{M} P(t) x(t)+P_{M} w(t) .
$$

Set $\eta(t)=P_{M} x(t), v(t)=P_{M} w(t)$, then $\eta(t), v(t) \in M$, and

$$
\begin{aligned}
& P_{M} P_{t} \eta_{t}=P_{M} P_{t} P_{M} x(t)=P_{M} P_{t}\left(I-\frac{1}{n} \mathbf{1} \cdot \mathbf{1}^{\tau}\right) x(t) \\
= & P_{M} P_{t} x(t)-P_{M} P_{t} \frac{1}{n} \mathbf{1} \cdot \mathbf{1}^{\tau} x(t)=P_{M} P_{t} x(t)-\frac{1}{n} P_{M} \mathbf{1} \cdot \mathbf{1}^{\tau} x(t) \\
= & P_{M} P_{t} x(t) .
\end{aligned}
$$

So system (5) is equivalent to

$$
\eta(t+1)=P_{M} P(t) \eta(t)+v(t),
$$

where $\eta(t), v(t) \in M$. Set $v=\{v(t)\}_{t=1}^{\infty}, U_{M}(\delta)=\{v:\|v(t)\| \leq \delta, v(t) \in M, \forall t \geq 1\}$. Here, system (14) is said to be robust stable on the subspace $M$, if for any $\eta(1) \in M$ and any $\varepsilon>0$, there exist constants $\delta=\delta(\varepsilon, \eta(1))>0, T=T(\varepsilon, \eta(1))>0$ such that $\sup _{v \in U_{M}(\delta)} \sup _{t>T}\|\eta(t)\| \leq \varepsilon$. Thus, robust consensus of system (5) has been transformed into the robust stability of system (14) on the subspace $M$.

We define $\Phi(k, i)$ as the state transition matrix of (14), that is

$$
\Phi(k+1, i)=P_{M} P(k) \Phi(k, i), \quad \Phi(i, i)=I, \quad \forall k \geq i \geq 0 .
$$

Then, we have

$$
\eta(t+1)=\Phi(t+1,0) \eta(0)+\sum_{i=1}^{t} \Phi(t+1, i+1) v(i) .
$$

To motivate further study, we introduce the following exponential stability lemma.

Lemma 2. Consider system (14) with $P(t)$ being stochastic matrix. If the system is robust stable on the subspace $M$, then there exist constants $N>0$ and $\lambda \in(0,1)$ such that

$$
\|\Phi(k+h, k)\| \leq N \lambda^{h}, \quad \forall k \geq 0, \quad \forall h \geq 1 .
$$

The proof is in Appendix 7.

The following lemma will establish the relationship between the exponential stability and the second smallest eigenvalue of $\sum L(t)$. 
Lemma 3. Consider system (14) with $P(t)=D^{-1}(t) A(t)$. Assume the weighted adjacency matrix $A(t)$ satisfies Assumption $\Lambda$ and is symmetric. If there exist constants $N>0$ and $\lambda \in(0,1)$ such that $\|\Phi(k+h, k)\| \leq N \lambda^{h}$ for any $k \geq 0, h \geq 0$. Then, there must exist a constant $q>0$ such that

$$
\inf _{k \geq 0} \lambda_{1}\left(\sum_{k}^{k+q-1} L(t)\right) \neq 0,
$$

where $L(t)=D(t)-A(t)$ is the Laplacian matrix.

In order to maintain the integrity of the context, the proof of this Lemma is also presented in Appendix 7.

Finally, by combining Lemma 2 with Lemma 3, we can finish the proof of (i) $\Rightarrow$ (ii).

3.2 The proof of (ii) $\Rightarrow$ (iii)

The following theorem provides the relationship between the connectivity of the graph and the eigenvalues of the weighted Laplacian matrix.

Theorem 2. Let $G$ be a graph and $L$ be its weighted Laplacian matrix. Then, the eigenvalue 0 of $L$ is simple if $G$ is connected. Moreover, if the algebraic multiplicity of the eigenvalue 0 of $L$ is $c$, then the graph $G$ has exactly c connected components.

Proof. The first part can be obtained from Lemma 13.9.1 of ref. [30]; The second part follows from the fact that the union of two disjoint graph has as its spectrum the union of the spectra of the original graphs.

Since $\sum_{k=t+1}^{k=t+q} L(k)$ is a weighted Laplacian matrix of the union graph of $\left\{G_{t+1}, G_{t+2}, \cdots, G_{t+q}\right\}$, by Theorem 2, we know that $\lambda_{1}\left(\sum_{k=t+1}^{k=t+q} L(k)\right) \neq 0$ is equivalent to the connectivity of the union graph. Thus the Proposition (ii) $\Rightarrow$ (iii) of Theorem 1 is true.

3.3 The proof of (iii) $\Rightarrow$ (i)

The union of graphs is closely related to the product of stochastic matrices. The following theorem provides a relationship between the connectivity of the union graph of $\left\{G_{t_{1}}, \cdots, G_{t_{m}}\right\}$ and the matrix products $P\left(t_{1}\right) P\left(t_{2}\right) \cdots P\left(t_{m}\right)$.

Lemma 4. [9] Let $\{P(t)\}$ be stochastic matrix with positive diagonal entries, and $G_{t}$ be the associated graph. If $\left\{G_{t_{1}}, \cdots, G_{t_{m}}\right\}$ is jointly connected, then the product of matrix $P\left(t_{1}\right) P\left(t_{2}\right) \cdots P\left(t_{m}\right)$ is SIA.

Let $\mathcal{P}$ be a matrix set. By a word (in the $\mathcal{P}$ ) of length $m$ we mean the product of $m \mathcal{P}^{\prime} s$ (cf. [27]). From the proof of Lemma 4 in ref. [27], we can see that the following result is also true.

Lemma 5. Let $\left\{P_{i}\right\}_{i=1}^{\infty}$ be a stochastic matrix sequence denoted by $\mathcal{P}$. If any word in the $\mathcal{P}^{\prime}$ s is SIA, then there exists a constant $T^{*}>0$ such that all words in the $\mathcal{P}^{\prime}$ s of length $\geq T^{*}$ are scrambling matrices, where $T^{*}$ only depends on the dimension of the matrix.

For a stochastic matrix $P=\left\{P_{i j}\right\}$, define

$$
\tau(P)=\frac{1}{2} \max _{i, j} \sum_{s=1}^{n}\left|P_{i s}-P_{j s}\right| .
$$


From ref. [29], we know that $\tau(P)=1-\lambda(P)$, and for any stochastic matrices $P(1)$ and $P(2)$, we have

$$
\tau(P(1) P(2)) \leq \tau(P(1)) \tau(P(2)) .
$$

Furthermore, the function $\tau(\cdot)$ also has the following property.

Lemma 6. [29] Let $y=\left[y_{1}, \cdots, y_{n}\right]^{\tau} \in \mathbf{R}^{n}$ be an arbitrary vector and $P=\left[P_{i j}\right]_{n \times n}$ be a stochastic matrix. If $z=P y, z=\left[z_{1}, \cdots, z_{n}\right]^{\tau}$, then we have

$$
\max _{s, s^{\prime}}\left|z_{s}-z_{s^{\prime}}\right| \leq \tau(P) \max _{j, j^{\prime}}\left|y_{j}-y_{j^{\prime}}\right| \text {. }
$$

We also need the following simple lemma, whose proof is in Appendix 7.

Lemma 7. Let $z \in \mathbf{R}^{n}, \Delta z=\max _{i, j}\left|z_{i}-z_{j}\right|, P_{M}$ be the projector onto $M$. Then

$$
\frac{\sqrt{2}}{2} \Delta z \leq\left\|P_{M} z\right\|=d(z, X) \leq \sqrt{n} \Delta z
$$

The proof of (iii) $\Rightarrow$ (i) :

For system (5), we define the state transition matrix as follows

$$
\Phi^{*}(k+1, i)=P(k) \Phi^{*}(k, i), \Phi^{*}(i, i)=I, \quad \forall k \geq i \geq 0,
$$

then we have

$$
x(t+1)=\Phi^{*}(t+1,0) x(0)+\sum_{i=1}^{t} \Phi^{*}(t+1, i+1) w(i) .
$$

By applying Lemma 6, we have

$$
\Delta x(t+1) \leq \tau\left(\Phi^{*}(t+1,0)\right) \Delta x(0)+\sum_{i=1}^{t} \tau\left(\Phi^{*}(t+1, i+1)\right) \Delta w(i) .
$$

From Lemma 4 , we know that $\Phi^{*}(t+q+1, t+1)$ is SIA for any $t$. Furthermore, by combining with Lemma 5, for any $t \geq 0$, we have

$$
\Phi^{*}(t+L, t)=\prod_{k=t}^{k=t+L-1} P(k) \text { is a scrambling matrix }
$$

where $L=q T^{*}$ is a constant. From (2) of Assumption $\Lambda$, we know that there exists a constant $\bar{\alpha}>0$ such that all the non-zero entries of $P(t)$ are larger than or equal to $\bar{\alpha}$. Then we have

$$
\lambda\left(\Phi^{*}(t+L, t)\right) \geq \bar{\alpha}^{L} .
$$

Hence

$$
\tau\left(\Phi^{*}(t+L, t)\right)=1-\lambda\left(\Phi^{*}(t+L, t)\right) \leq 1-\bar{\alpha}^{L}=\sigma, \quad \forall t \geq 0 .
$$

For any $t \geq 0$ and $h \geq 0$, there exists an integer $k_{0} \geq 0$ such that $k_{0} L<h \leq\left(k_{0}+1\right) L$. By (19), we have

$$
\begin{aligned}
\tau\left(\Phi^{*}(t+h, t)\right) & \leq \tau\left(\Phi^{*}\left(t+h, t+k_{0} L\right)\right) \cdot \tau\left(\Phi^{*}\left(t+k_{0} L, t+\left(k_{0}-1\right) L\right) \cdots \Phi^{*}(t+L, t)\right) \\
& \leq \sigma^{k_{0}} \leq \sigma^{\frac{h}{L}-1}=\sigma^{-1}\left(\sigma^{\frac{1}{L}}\right)^{h} .
\end{aligned}
$$


Define $N=\sigma^{-1}, \lambda=\sigma^{\frac{1}{t}}$. Then $0<\lambda<1$ and

$$
\tau\left(\Phi^{*}(t+h, t)\right) \leq N \lambda^{h}, \quad \forall t \geq 0, h \geq 0,
$$

where $N$ and $\lambda$ are independent of $t$ and $h$. From (21), we have

$$
\lim _{t \rightarrow \infty} \tau\left(\Phi^{*}(t+1,0)\right) \leq \lim _{t \rightarrow \infty} N \lambda^{t+1}=0 .
$$

Moreover, for any $t \geq 0$, we have

$$
\sum_{i=1}^{t} \Phi^{*}(t+1, i+1) \leq \sum_{i=1}^{t} N \lambda^{t-i}<\frac{N}{1-\lambda} .
$$

Thus

$$
\sup _{t \geq 0} \sum_{i=1}^{t} \Phi^{*}(t+1, i+1) \leq \frac{N}{1-\lambda} .
$$

By (22), for any $\delta>0$, there exists $T>0$ such that

$$
\tau\left(\Phi^{*}(t, 0)\right) \leq \frac{\delta}{\Delta x_{0}}, \quad \forall t \geq T
$$

For any $\{w(t)\}$ satisfying $\sup _{t \geq 0} d(w(t), X) \leq \delta$, by Lemma 7, we have $\sup _{t \geq 0} \Delta w(t) \leq \sqrt{2} \delta$, which, in conjunction with (23) and (24), yields

$$
\Delta x(t) \leq \delta+\frac{\sqrt{2} N}{1-\lambda} \delta=\left(1+\frac{\sqrt{2} N}{1-\lambda}\right) \delta, \quad \forall t \geq T .
$$

By taking $f(s)=\sqrt{n}\left(1+\frac{\sqrt{2} N}{1-\lambda}\right) s$, obviously we have $f(\cdot) \in K_{0}$, and

$$
d(x(t), X) \leq f(\delta), \quad \forall t \geq T .
$$

Thus, we complete the proof of (iii) $\Rightarrow$ (i) of Theorem 1 .

Remark 1. From Lemma 2, we know that the exponential stability of the projected system (14) is essential for the robust consensus. However, to the best of our knowledge, almost all the existing results only analyzed the asymptotic stability of the projected system, which might not be powerful enough for dealing with the influence of noise.

\section{Directed information exchange topology}

In this section, we will generalize the above results to the case of directed information exchange. The information exchange with directed topology can be found in many biological, social, and engineering systems, such as the "leader-follower" model where the leader can influence the followers while the followers can not influence the leader.

The main result of this section are summarized in the following theorem.

Theorem 3. Consider the system (4) under Assumption A. Then it is robust consensus if and only if there exists a constant $q>0$ such that for any $t \geq 0$ the union of neighbor digraphs $\left\{G_{t+1}, G_{t+2}, \cdots, G_{t+q}\right\}$ has a spanning tree. 
To prove the main result, we need the following lemmas which is about the algebraic multiplicity of the eigenvalue 1 of stochastic matrix.

Lemma 8. Let $P$ be a stochastic matrix with $P_{M}$ being its projector onto $M$. If $\rho\left(P_{M} P\right)<1$, then 1 is a simple eigenvalue of $P$, where $\rho(A)$ is the spectral radius of a matrix $A$.

Proof From the theorem of finite dimensional Markov chain (see [32]), we know that there exists a matrix $K$ such that

$$
\lim _{n \rightarrow \infty} \frac{1}{n} \sum_{i=1}^{n} P^{i}=K
$$

Thus

$$
\lim _{n \rightarrow \infty} P_{M} \frac{1}{n} \sum_{i=1}^{n} P^{i}=P_{M} K
$$

On account of $\rho\left(P_{M} P\right)<1$, there exists a matrix norm $\|\cdot\|_{l}$ such that

$$
\left\|P_{M} P\right\|_{l} \doteq \mu<1 .
$$

By (11),

$$
\left\|P_{M} \frac{1}{n} \sum_{i=1}^{n} P^{i}\right\|_{l}=\left\|\frac{1}{n} \sum_{i=1}^{n}\left(P_{M} P\right)^{i}\right\|_{l} \leq \frac{1}{n} \sum_{i=1}^{n}\left\|P_{M} P\right\|_{l}^{i} \leq \frac{1}{n} \sum_{i=1}^{n} \mu^{i}
$$

Thus

$$
\begin{gathered}
\lim _{n \rightarrow \infty}\left\|\frac{1}{n} \sum_{i=1}^{n}\left(P_{M} P\right)^{i}\right\|_{l}=0, \\
P_{M} K=\lim _{n \rightarrow \infty} \frac{1}{n} \sum_{i=1}^{n}\left(P_{M} P\right)^{i}=0 .
\end{gathered}
$$

Since the rank of $P_{M}$ is $n-1$, the rank of $K$ is not greater than 1 .

1 is an eigenvalue of $P$ and $\rho(P) \leq 1$. Now, we prove 1 is simple. If not, from the Jordan canonical form, we have a nonsingular matrix $T$ such that

$$
P=T\left(\begin{array}{ccccc}
\lambda_{1} & 0 & 0 & \cdots & 0 \\
* & \lambda_{2} & 0 & \cdots & 0 \\
0 & * & \lambda_{3} & \cdots & 0 \\
\cdots & \cdots \cdots & \cdots & \cdots & \cdots \\
0 & \cdots & 0 & * & \lambda_{n}
\end{array}\right) T^{-1}
$$

where $\left|\lambda_{i}\right| \leq 1$ and $\lambda_{1}=\lambda_{2}=\cdots=\lambda_{k}=1$ with $k \geq 2$. Thus

$$
\lim _{n \rightarrow \infty} \frac{1}{n} \sum_{i=1}^{n} P^{i}=T\left(\begin{array}{ccccc}
1 & 0 & 0 & \cdots & 0 \\
* & 1 & 0 & \cdots & 0 \\
* & * & * & \cdots & 0 \\
\ldots & \ldots & \ldots & \ldots & \ldots \\
* & \cdots & * & * & *
\end{array}\right) T^{-1}
$$

which implies that the rank of $K$ is larger than 1 . This is a contradiction, so 1 is a simple eigenvalue of $P$.

Lemma 9. [13] For a stochastic matrix $P$, the eigenvalue 1 is simple if and only if its associated digraph $\Gamma(P)$ has a spanning tree. 
Lemma 10. [13] Let $\{P(t)\}$ be a sequence of stochastic matrix with positive diagonal entries, and with associated digraphs denoted by $G_{t}$. If for any sequence $\left\{i_{1}, i_{2}, \cdots, i_{m}\right\}$, the union of the directed graphs $\left\{G_{i_{1}}, \cdots, G_{i_{m}}\right\}$ has a spanning tree, then the matrix product $P\left(i_{m}\right) \cdots P\left(i_{2}\right) P\left(i_{1}\right)$ is $S I A$.

\section{Proof of Theorem 3:}

Sufficiency: By using Lemma 10, and following the same analysis in the proof of (iii) $\Rightarrow$ (i) of Theorem 1, one can get the desired conclusion directly.

Necessity: As we have seen in the above section, the robust consensus of the system (5) can be transformed to the stability of the system (14) on the subspace $M$. Define

$$
P^{(t, r)}=P(t+r-1) \cdots P(t+1) P(t),
$$

from Lemma 2, we know that for any $t \geq 0$ there exists a constant integer $q$ such that

$$
\left\|P_{M} P^{(t, q)}\right\|<1
$$

Let $P^{(t)}$ denote $P^{(t, q)}, D^{(t)}$ denote the associated directed graph of $P^{(t)}, G_{k}$ denote the neighbor graph associated with $P(k), G^{(t)}$ denote the union of digraphs $\left\{G_{t}, G_{t+1}, \cdots, G_{t+q}\right\}$.

From Lemma 8 and (25), we know 1 is a simple eigenvalue of $P^{(t)}$. Furthermore, the digraph $D^{(t)}$ has a spanning tree according to Lemma 9. To complete the proof of the sufficiency part of Theorem 3, we need to show that $G^{(t)}$ has a spanning tree.

Now, let vertex $r$ be the root of the graph $D^{(t)}$, then for any other vertex $j \in V$, there is a $r \rightarrow j$ path in digraph $D^{(t)}$, i.e. there is a sequence of $\operatorname{arcs}\left(r, i_{1}\right),\left(i_{1}, i_{2}\right),\left(i_{2}, i_{3}\right), \cdots$, $\left(i_{m-2}, i_{m-1}\right),\left(i_{m-1}, j\right)$ in $D^{(t)}$ connecting $r$ to $j$. By the relations between $D^{(t)}$ and $P^{(t)}$, we know that the following elements of $P^{(t)}: P_{i_{1}, r}^{(t)}, P_{i_{2}, i_{1}}^{(t)}, \cdots, P_{i_{m-1}, i_{m-2}}^{(t)}, P_{j, i_{m-1}}^{(t)}$ are all nonzero. Now, we consider each nonzero entry $P_{i_{s+1}, i_{s}}^{(t)}, s=0,1, \cdots, m-1\left(i_{0}\right.$ denotes $r, i_{m}$ denotes $\left.j\right)$, by using the property of matrix product, we know that there exists a nonzero item $P_{i_{s+1}, k_{q-1}}(t+q-$ 1) $P_{k_{q-1}, k_{q-2}}(t+q-2) \cdots P_{k_{1}, i_{s}}(t)$ for some $k_{1}, k_{2}, \cdots, k_{q-1}$. Note that $P_{i, j}(k) \neq 0$ means there is an $\operatorname{arc}(j, i)$ in digraph $G_{k}$, thus the item $P_{i_{s+1}, k_{q-1}}(t+q-1) P_{k_{q-1}, k_{q-2}}(t+q-2) \cdots P_{k_{1}, i_{s}}(t) \neq 0$ means there is a path in the union digraph $G^{(t)}$ connecting $i_{s}$ with $i_{s+1}$. Hence, for each nonzero entry $P_{i_{s+1}, i_{s}}^{(t)}, s=0,1, \cdots, m-1$, there is a path $i_{s} \rightarrow i_{s+1}$ in digraph $G^{(t)}$, and so it is obvious that there is a path in digraph $G^{(t)}$ connecting $r$ with $j$. According to the arbitrariness of vertex $j$, we know that the digraph $G^{(t)}$ has a spanning tree rooted at $r$, i.e. the union of neighbor digraphs $\left\{G_{t}, G_{t+1}, \cdots, G_{t+q-1}\right\}$ has a spanning tree. The proof of Theorem 3 is complete.

\section{Simulations}

In this section, we consider the consensus behavior of four agents with switching topologies. Three representative examples are given for illustration. In the simulations, the initial states of the agents are chosen randomly from $[0,3]$, the adjacency matrix $A(t)$ associated with the network topology is $0-1$ matrix, i.e. $a_{i j}(t)=1$ iff $j \in N_{i}(t)$ and otherwise $a_{i j}(t)=0$. We assume each agent treats itself as a neighbor, and can access its own information accurately at any time. That is $x_{i}(t+1)=x_{i}(t)$ if agent $i$ has no other neighbors except itself at time $t$. 
Example 1. Consider a dynamical network of four agents with undirected information exchange topologies. The agent applies the consensus protocol (4). The information exchange topologies are changed as follows: when $t=3 k, k=0,1, \cdots$, it has the structure as shown in Figure 1.(a), that is $a_{12}=a_{21}=1$; when $t=3 k+1$, it is described by Figure 1.(b); when $t=3 k+2$, it is Figure 1.(c).

$\left.\right|_{\text {A2 }} ^{\text {A1 }}$

(a)
(A4)

(A3)

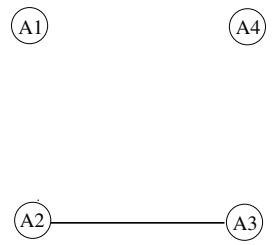

(b)
(A1)

(A2)

(c)

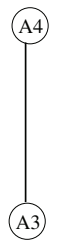

(A3)

Fig. 1. Three topologies of undirected information exchange.

Figures 2.(a) and (b) show the evolution of protocol (4) with noise chosen randomly from $[-0.5,0.5]$. Figure 2.(a) depicts the information states $x(i), i=1,2,3,4$. Figure 2.(b) depicts the maximal difference of information states indexed by $\max _{i j}\left|x_{i}-x_{j}\right|$. Figure 2.(c) shows the relationship between the difference of the states and the intensity of the noise, where the point represent the mean difference of the states over one running indexed by $\frac{1}{T} \sum_{t=1}^{T} \max _{i j} \mid x_{i}(t)-$ $x_{j}(t) \mid$ with $T=50$ in the simulations, and the range of the noise add 0.1 at each running. Figure 2.(c) shows that the higher the noise intensity is, the higher the difference of the states is. From Figure (2), we can see that the system in Example 1 is robust consensus.

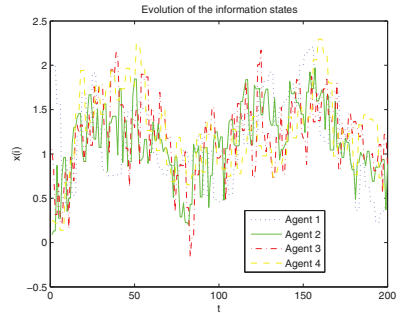

(a)

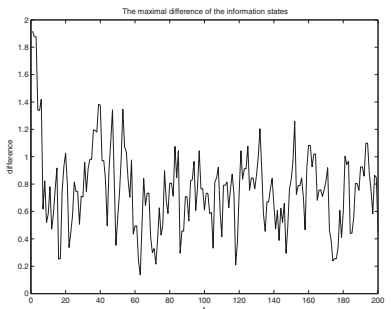

(b)

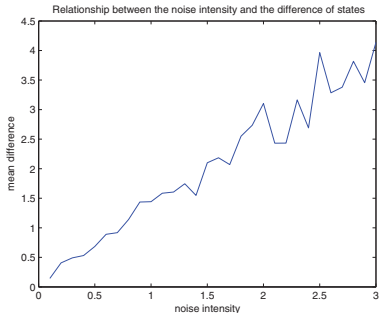

(c)

Fig. 2. Evolution of the protocol (4) with the undirected topologies given by Figure 1.

Example 2. Consider a dynamical network of four agents with directed information exchange topologies as shown in Figure 3. The agent applies the consensus protocol (4). When $t=3 k$, $k=0,1, \cdots$, the information exchange topology has the structure as shown in Figure 3.(a), that is $a_{21}=1$ and others equal zero; when $t=3 k+1$, it is described by Figure 3.(b); when $t=3 k+2$, it is Figure 3.(c).

Figure 4 shows the evolution of the protocol (4) with the above directed topologies. Figures 4.(a),(b) and (c) show the same items as those in Figures 2.(a),(b) and (c). In view of Figure 3, agent 1 does not have other neighbors except itself during the evolution. Thus, in Figure 4.(a), agent 1 keep its own state all the time, and other agents move near it. 


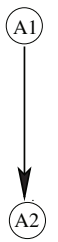

(a)
(A4)

(A3)

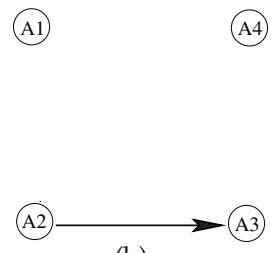

(b)
(A1)

(A2)

(c)

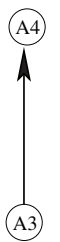

(A3)

Fig. 3. Three topologies of directed information exchange.

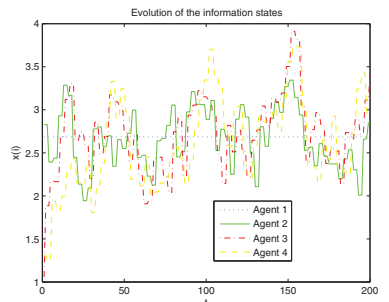

(a)

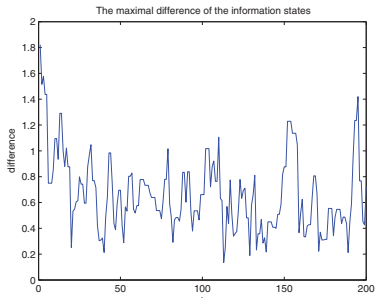

(b)

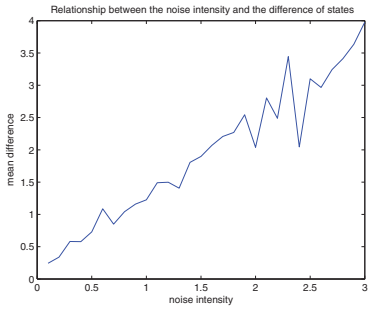

(c)

Fig. 4. Evolution of the protocol (4) with the directed topologies given by Figure 3.

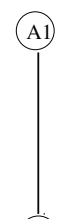

(A2)

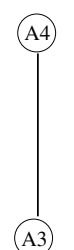

(A3)

(a)

(A1)

(A4)

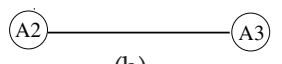

(b)

Fig. 5. Two topologies of undirected information exchange.

Example 3. In this example, we will present two experiments to show that the periodical connectivity of the neighbor graphs is essential for the robust consensus of the agents. In the following experiments, the four agents apply the consensus protocol (4) with the noise chosen randomly from $[-0.3,0.3]$, and the neighbor graphs have the topologies as shown in Figure 5.

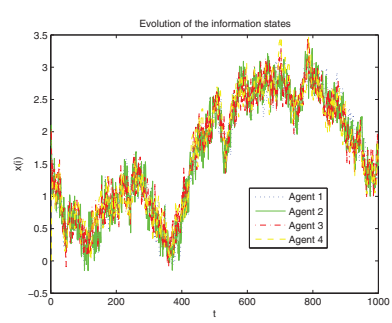

(a)

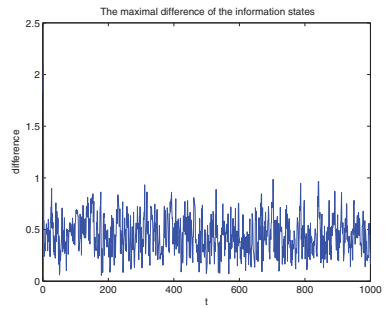

(b)

Fig. 6. Evolution with periodically connected information exchange topologies. 
In the first experiment, the dynamics of the information exchange topology is defined as follows: when $t=2 k, k=0,1, \cdots$, the topology has the structure as shown in Figure 5.(a), that is $a_{12}=a_{21}=1$ and $a_{34}=a_{43}=1$; when $t=2 k+1$, it has the structure as shown in Figure 5.(b). Figure 6.(a) shows the information states $x(i)$, and Figure 6.(b) depicts the maximal difference of the information states. From these figures, we can see that the system is robust consensus.

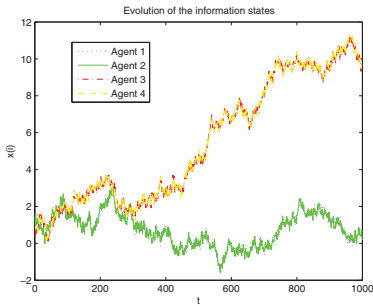

(a)

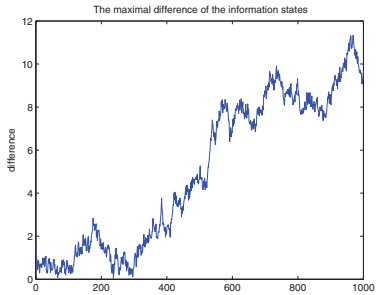

(b)

Fig. 7. Evolution with infinitely connected information exchange topologies.

The second experiment is carried out as follows: when $t=2^{k}, k=0,1, \cdots$, the information exchange topology has the structure as shown in Figure 5.(b), otherwise it has the structure as shown in Figure 5.(a). Therefore, for any time $T$, the union graph of the neighbor graphs $\left\{G_{t}: t \geq T\right\}$ is connected (called infinitely connected for short). However, from Figure 7 we know that the infinite connectivity is not enough for the robust consensus.

\section{Conclusions}

This paper studies the robust consensus of multi-agent systems with bounded noises. The information exchange topologies are time-varying. For the case of undirected topologies, the equivalence among the robust consensus, the positivity of the second smallest eigenvalue of the Laplacian matrix, and the periodically joint connectivity of the neighbor graphs are established. For the case of directed topologies, the robust consensus can be achieved if and only if there exists a spanning tree in the union of the neighbor graph periodically. From the theoretical analysis and the simulation results, we can see that the periodical connectivity of the neighbor graphs is essential for the robust consensus of the agents.

\section{Appendix}

To prove Lemma 2, we need the following theorem.

Theorem 4. [28] Consider the following time-varying system:

$$
y_{k+1}=B_{k} y_{k}, \quad k \geq 0,
$$

where $B_{k} \in \mathbf{R}^{n \times n}$ and $y_{k} \in \mathbf{R}^{n}$. Let $\Psi(k, i)$ be the state transition matrix of system $(26): \Psi(k+1, i)=$ $B_{k} \Psi(k, i), \Psi(i, i)=I, \forall k \geq i \geq 0$. Then system (26) is exponentially stable in the sense that there exist constants $M_{0}>0$ and $c>0$ such that

$$
\|\Psi(k+h, k)\| \leq M_{0} \exp \{-c h\} \forall k \geq 0, \forall h \geq 0,
$$

if and only if $\sum_{i=1}^{k}\|\Psi(k, i)\| \leq M_{1}, \forall k \geq 0$, where $M_{1}$ is independent of $k$. 
Proof of Lemma 2 Set $v=\left\{v_{t}\right\}_{t=1}^{\infty}, U=\left\{v:\left\|v_{t}\right\| \leq \delta_{0}, v(t) \in M, \forall t \geq 1\right\}$. According to the assumption, we know that there exist $f\left(\delta_{0}\right)>0, T_{0}>0$ such that for any $v \in U$ and $\eta_{0}=0$, $\left\|\eta_{t}\right\| \leq f\left(\delta_{0}\right), t \geq T_{0}$. By (16), for any $v \in U$, we have

$$
\sup _{t \geq T_{0}-1}\left\|\sum_{i=1}^{t} \Phi(t+1, i+1) v_{i}\right\| \leq f\left(\delta_{0}\right)=\varepsilon_{0}
$$

We want to prove that there exists a constant $N_{1}>0$ such that for any $v \in U$

$$
\sup _{t \geq T_{0}-1} \sum_{i=1}^{t}\left\|\Phi(t+1, i+1) v_{i}\right\| \leq N_{1}
$$

We will prove this by reduction to absurdity. Assume that there exist $t_{0} \geq T_{0}-1$ and $u \in U$ such that $\sum_{i=1}^{t_{0}}\left\|\Phi\left(t_{0}+1, i+1\right) u_{i}\right\| \geq 2 n \varepsilon_{0}$, where $n$ is the dimension of space. Let $\Gamma_{i}(s, j)$ be the $(s, j)$ entry of matrix $\Phi\left(t_{0}, i+1\right)$, and let $u_{i}(j)$ be the $j$ th element of $u_{i}$. Then there must exist an integer $s \in[1, n]$ such that

$$
\sum_{i=1}^{t_{0}}\left|\sum_{j=1}^{n} \Gamma_{i}(s, j) u_{i}(j)\right| \geq 2 \varepsilon_{0}
$$

Define $\hat{u}_{i}(j)=\left|u_{i}(j)\right| \operatorname{sgn}\left\{\Gamma_{i}(s, j)\right\}, 1 \leq j \leq n$, where $\operatorname{sgn}(\cdot)$ is the symbolic function. Obviously sup $\sup _{t \geq 0}\left\|\hat{u}_{t}\right\|=\sup _{t \geq 0}\|u(t)\| \leq \delta_{0}$. By (29), we have

$$
\left\|\sum_{i=1}^{t_{0}} \Phi\left(t_{0}+1, i+1\right) \hat{u}_{i}\right\| \geq\left|\sum_{i=1}^{t_{0}} \sum_{j=1}^{n} \Gamma_{i}(s, j) \hat{u}_{i}(j)\right|=\sum_{i=1}^{t_{0}} \sum_{j=1}^{n}\left|\Gamma_{i}(s, j)\right|\left|u_{i}(j)\right| \geq 2 \varepsilon_{0} .
$$

Decompose $\left\{\hat{u}_{i}, i=1, \cdots, t_{0}\right\}$ into two orthogonal vectors $\alpha_{i}$ and $\beta_{i}$, such that $\hat{u}_{i}=\alpha_{i}+$ $\beta_{i}, \alpha_{i} \in M, \beta_{i} \in X$. Then $\left\|\hat{u}_{i}\right\|=\left\|\alpha_{i}\right\|+\left\|\beta_{i}\right\| ;$ thus $\left\|\alpha_{i}\right\| \leq\left\|\hat{u}_{i}\right\|$. Noting that $P(t)$ is stochastic, we have $P_{M} P(t) \beta_{i}=0, \forall t \geq 0, \forall i \in\left[1, t_{0}\right]$. Thus by (30), we have

$\left\|\sum_{i=1}^{t_{0}} \Phi\left(t_{0}+1, i+1\right) \alpha_{i}\right\|=\left\|\sum_{i=1}^{t_{0}} \Phi\left(t_{0}+1, i+1\right)\left(\hat{u}_{i}-\beta_{i}\right)\right\|=\left\|\sum_{i=1}^{t_{0}} \Phi\left(t_{0}+1, i+1\right) \hat{u}_{i}\right\| \geq 2 \varepsilon_{0}$.

Now define a new sequence as

$$
u^{*}= \begin{cases}\alpha_{i}, & i \in\left[1, t_{0}\right] \\ u_{i}, & i>t_{0}\end{cases}
$$

Obviously $u^{*} \in U$, and by (31), we have

$$
\sup _{t \geq T_{0}-1}\left\|\sum_{i=1}^{t} \Phi(t+1, i+1) u_{i}^{*}\right\| \geq\left\|\sum_{i=1}^{t_{0}} \Phi\left(t_{0}+1, i+1\right) \alpha_{i}\right\| \geq 2 \varepsilon_{0} .
$$

It is opposite to (27). Thus (28) holds.

For any vector $\xi \in \mathbf{R}^{n}$ with $\|\xi\| \leq \delta_{0}$, do orthogonal decomposition on $\xi: \xi=\xi_{1}+\xi_{2}, \xi_{1} \in$ $M, \xi_{2} \in X$. Then $\|\xi\|=\left\|\xi_{1}\right\|+\left\|\xi_{2}\right\|,\left\|\xi_{1}\right\| \leq \delta_{0}$, and $P_{M} P(t) \xi_{2}=0, \forall t \geq 0$. By (28), we have

$$
\sup _{t \geq T_{0}-1} \sum_{i=1}^{t}\|\Phi(t+1, i+1) \xi\|=\sup _{t \geq T_{0}-1} \sum_{i=1}^{t}\left\|\Phi(t+1, i+1) \xi_{1}\right\| \leq N_{1} .
$$


On account of the arbitrariness of $\xi$, there must exist a constant $N_{2}>0$ such that

$$
\sup _{t \geq T_{0}-1} \sum_{i=1}^{t}\|\Phi(t+1, i+1)\| \leq N_{2}
$$

Because $\{P(t)\}_{t=1}^{t=T_{0}}, P_{M}$ and $T_{0}$ are bounded, we can choose a constant $N_{3}>0$ such that

$$
\sup _{t \leq T_{0}-1} \sum_{i=1}^{t}\|\Phi(t+1, i+1)\| \leq N_{3} .
$$

Taking $\bar{N}=\max \left\{N_{2}, N_{3}\right\}$ which is independent of $t$, by (32)(33), we have $\sum_{i=1}^{t}\|\Phi(t, i)\| \leq$ $\bar{N}, \forall t \geq 0$. According to Theorem 4 , there exist constants $N>0$ and $\lambda \in(0,1)$ such that $\|\Phi(k+h, k)\| \leq N \lambda^{h}, \forall k \geq 0, \forall h \geq 1$.

Proof of Lemma 3 According to the assumptions of this lemma, we can choose an integer $q>0$ such that

$$
\|\Phi(k+q, k)\|=\left\|P_{M} \prod_{t=k}^{k+q-1} P(t)\right\| \leq \frac{1}{2}
$$

Let $\rho_{k}=\lambda_{1}\left(\sum_{k}^{k+q-1} L(t)\right)$ and $x_{k}^{*}$ be the corresponding unit eigenvector. Then we have $\rho_{k}=$ $\sum_{k}^{k+q-1} x_{k}^{* \tau} L(t) x_{k}^{*}$. Since $L(t) \cdot \mathbf{1}=0$ for all $t \geq 1, x_{k}^{*}$ can be chosen from the subspace $M$.

For any integers $t_{j} \in[k, k+q-1], j=1, \cdots, s \leq q$, we have

$$
\begin{aligned}
& x_{k}^{* \tau} P_{M} D^{-1}\left(t_{1}\right) L\left(t_{1}\right) \cdots D^{-1}\left(t_{s}\right) L\left(t_{s}\right) x_{k}^{*} \\
\leq & \left\|x_{k}^{* \tau} P_{M} D^{-1}\left(t_{1}\right) L\left(t_{1}\right) \cdots D^{-1}\left(t_{s}\right) L\left(t_{s}\right)^{\frac{1}{2}}\right\| \cdot\left\|L\left(t_{s}\right)^{\frac{1}{2}} x_{k}^{*}\right\| \\
\leq & \beta^{2 q}\left(x_{k}^{* \tau} L\left(t_{s}\right) x_{k}^{*}\right)^{\frac{1}{2}} \leq \beta^{2 q} \rho_{k}^{\frac{1}{2}} .
\end{aligned}
$$

Let $b$ be the uniform upper bound of $\|L(t)\|$ and $\left\|D^{-1}(t)\right\|$ in view of Assumption $\Lambda$. From (34),(35) and the Schwarz inequality, it follows that

$$
\begin{aligned}
\frac{1}{2} & \geq\|\Phi(k+q, k)\|=\left\|P_{M} \prod_{t=k}^{k+q-1} P(t)\right\|=\left\|P_{M} \prod_{t=k}^{t=k+q-1}\left(I-D^{-1}(t) L(t)\right)\right\| \\
& \geq x_{k}^{* \tau} P_{M} \prod_{t=k}^{t=k+q-1}\left(I-D^{-1}(t) L(t)\right) x_{k}^{*} \\
& =1-\sum_{s=1}^{q} \sum_{k \leq t_{1} \leq \cdots \leq t_{s} \leq k+q-1} x_{k}^{* \tau} P_{M} D^{-1}\left(t_{1}\right) L\left(t_{1}\right) \cdots D^{-1}\left(t_{s}\right) L\left(t_{s}\right) x_{k}^{*} \\
& \geq 1-\sum_{s=1}^{q}\left(\begin{array}{l}
q \\
s
\end{array}\right) \beta^{2 q} \rho_{k}^{\frac{1}{2}} .
\end{aligned}
$$

So, $\rho_{k}^{\frac{1}{2}} \geq \frac{1}{2 \sum_{s=1}^{q}\left(\begin{array}{l}q \\ s\end{array}\right) \beta^{2 q}}, \quad \forall k \geq 0$, which means that $\inf _{k \geq 0} \lambda_{1}\left(\sum_{k}^{k+q-1} L(t)\right) \neq 0$. Thus Lemma 3 is true. 
Proof of Lemma 4 On the one hand, from $\min _{i} z_{i} \leq \frac{\sum_{i} z_{i}}{n} \leq \max _{i} z_{i}$, we have, for any $z_{j}$,

$$
z_{j}-\max _{i} z_{i} \leq z_{j}-\frac{\sum_{i} z_{i}}{n} \leq z_{j}-\min _{i} z_{i} .
$$

So, $\left|z_{j}-\frac{\sum_{i} z_{i}}{n}\right| \leq \max \left\{\max _{i} z_{i}-z_{j}, z_{j}-\min _{i} z_{i}\right\} \leq \Delta z$. Thus

$$
\left\|P_{M} z\right\|=\left\|P_{M}\left(z-\frac{\mathbf{1}^{\tau} z \mathbf{1}}{n}\right)\right\|=\left\|z-\frac{\mathbf{1}^{\tau} z \mathbf{1}}{n}\right\| \leq \sqrt{n} \Delta z .
$$

On the other hand,

$$
\begin{aligned}
\left|z_{i}-z_{j}\right| & =\left|\left(z_{i}-\frac{\sum_{s} z_{s}}{n}\right)-\left(z_{j}-\frac{\sum_{s} z_{s}}{n}\right)\right| \leq\left|z_{i}-\frac{\sum_{s} z_{s}}{n}\right|+\left|z_{j}-\frac{\sum_{s} z_{s}}{n}\right| \\
& \leq \sqrt{2} \sqrt{\left(z_{i}-\frac{\sum_{s} z_{s}}{n}\right)^{2}+\left(z_{j}-\frac{\sum_{s}}{n}\right)^{2}} \leq \sqrt{2}\left\|z-\frac{\mathbf{1}^{\tau} z \mathbf{1}}{n}\right\|=\sqrt{2}\left\|P_{M} z\right\|,
\end{aligned}
$$

Thus, $\Delta z \leq \sqrt{2}\left\|P_{M} z\right\|$. From (9) and the above analysis, it follows that

$$
\frac{\sqrt{2}}{2} \Delta z \leq\left\|P_{M} z\right\|=d(z, X) \leq \sqrt{n} \Delta z .
$$

\section{Acknowledgements}

This work was supported by the NSF of P. R. China under Grant Nos. 60804043, and a grant from the Major State Basic Research Development Program of China (973 Program) (No. 2010CB731400).

\section{References}

[1] De Groot, M.H. (1974). Reaching a consensus, J. Amer. Statist. Assoc., Vol.69, 118-121

[2] Chatterjee, \& S., Seneta, E. (1977). Towards consensus: some convergence theorems on repeated averaging, J. Appl. Prob, Vol.14, 89-97

[3] Fischer M. J. (1983). The consensus problem in unreliable distributed systems (a brief survey), In Foundations of Computation Theory, M. Karpinsky ed., Lecture Notes in Comput, 127-140, Springer-Verlag, New York

[4] Lynch, N. (1996). Distributed Algorithms, Morgan Kaufmann, San Mateo, CA

[5] Vicsek, T., Czirok, A., Ben Jacob, I., Cohen, I., \& Schochet, O. (1995). Novel type of phase transitions in a system of self-driven particles, Phys. Rev. Lett., Vol.75, 1226-1229

[6] Visscher, P. K. (2003). How self-organization evolves, Nature, Vol.421, 799-800

[7] Shamma, J. (2008). Cooperative Control of Distributed Multi-Agent Systems, Wiley-Interscience, New York

[8] Ren, W., \& Beard, R.W. (2008). Distributed Consensus in Multi-vehicle Cooperative Control, Communications and Control Engineering Series, Springer-Verlag, London

[9] Jadbabaie, A., Lin, J., \& Morse, A.S. (2003). Coordination of groups of mobile autonomous agents using nearest neighbor rules, IEEE Trans. Auto. Contr., Vol.48, No.6, 988-1001 
[10] Liu, Z.X., Han, J., \& Guo, L. (2006). Analysis and Regulation of Dynamical Networks Based on Vicsek's Model, Complex Networks, Guo, L. \& Xu, X.X.(Ed.), 49-66, Shanghai Scientific \& Technological Education press, Shanghai

[11] Ma, H. B. (2009). Decentralized Adaptive Synchronization of a Stochastic Discrete-Time Multiagent Dynamic Model, SIAM J. Control Optim., Vol. 48, 859-880

[12] Cao, M., Morse, A.S., \& Anderson, B.D.O. (2008). Reaching a Consensus in a dynamically changing environment: a graphical approach, SIAM Journal on Control and Optimization, Vol.47, 575-600

[13] Ren, W., \& Beard, R. W. (2005). Consensus seeking in multi-agent systems under dynamically changing interaction topologies, IEEE Trans. Auto. Contr., Vol.50, No.5, 655-661

[14] Moreau, L.(2005). Stability of multi-agent systems with time-dependent communication links, IEEE Trans. Auto. Contr., Vol.50, No.2, 169-182

[15] Hong, Y., Gao, L., Cheng, D., \& Hu, J. (2007). Lyapunov-based approach to multi-agent systems with switching jointly-connected interconnection, IEEE Trans. Auto. Contr., vol.52, 943-948

[16] Olfati-Saber, R. (2006). Flocking for multi-agent dynamic systems: algorithms and theory, IEEE Trans. Auto. Contr., Vol.51, 401-420

[17] Su, H., Wang, X., \& Lin, Z. (2009). Flocking of multi-agents with a virtual leader, IEEE Trans. Auto. Contr., Vol.54, 293-307

[18] Tahbaz-Salehi, A., \& Jadbabaie, A. (2008). A necessary and sufficient condition for consensus over random networks, IEEE Trans. Auto. Contr., Vol.53, No.3, 791-795

[19] Hatano, Y., \& Mesbahi, M. (2005). Agreement over random network, IEEE Trans. Auto. Contr., Vol.50, No.11, 1867-1872

[20] Olfati-Saber, R., \& Murray, R. M. (2004). Consensus problems in networks of agents with switching topology and time-delays, IEEE Trans. Auto. Contr., Vol.49, 1520-1533

[21] Tian, Y., \& Liu, C. (2008). Consensus of multi-agent systems with diverse input and communication delays, IEEE Trans. Auto. Contr., Vol.53, No.9, 2122-2128

[22] Xiao, L., Boyd, S., \& Kim, S.J. (2007). Distributed average consensus with least-mean square deviation, Journal of Paralell and Distributed Computing, Vol.67, No.1, 33-46

[23] Huang, M., \& Manton, J. H. (2009). Coordination and consensus of networked agents with noisy measurement: stochastic algorithms and asymptotic behavior, SIAM Journal on Control and Optimization, Vol.48, No.1, 134-161

[24] Li, T., \& Zhang, J.F. (2010). Consensus conditions of multi-agent systems with time-varying topologies and stochastic communication noises, IEEE Trans. Auto. Contr., Vol.55, No.9, 2043-2057

[25] Wang, L., \& Liu, Z.X. (2009). Robust consensus of multi-agent systems with noise, Science in China, Vol.52, No.5, 824-834

[26] Wang, L., \& Guo, L. (2008). Robust consensus and soft control of multi-agent systems with noises, Journal of Systems Science and Complexity, Vol.21, 406-415

[27] Wolfowitz, J. (1963). Products of indecomposable, aperiodic, stochastic matrices, Proceedings of the American Mathematical Society, Vol.15, 733-737

[28] Guo, L. (1993). Time-Varying Stochastic Systems, Jilin Science and Technology Press, Jilin

[29] Seneta, E. (1984). Non-negative Matrices and Markov Chains( 2nd ed.), Springer, New York

[30] Godsil, C., \& Royle, G. (2001). Algebraic Graph Theory, Springer-Verlag, New York

[31] Horn, R. A., \& Johnson, C. R. (1985). Matrix Analysis, Cambridge University, New York

[32] Doob, J. L. (1953). Stochastic Processes, John Wiley, New York 


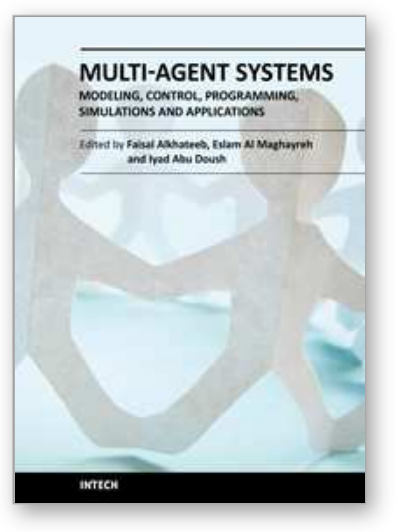

\author{
Multi-Agent Systems - Modeling, Control, Programming, \\ Simulations and Applications \\ Edited by Dr. Faisal Alkhateeb
}

ISBN 978-953-307-174-9

Hard cover, 522 pages

Publisher InTech

Published online 01, April, 2011

Published in print edition April, 2011

A multi-agent system (MAS) is a system composed of multiple interacting intelligent agents. Multi-agent systems can be used to solve problems which are difficult or impossible for an individual agent or monolithic system to solve. Agent systems are open and extensible systems that allow for the deployment of autonomous and proactive software components. Multi-agent systems have been brought up and used in several application domains.

\title{
How to reference
}

In order to correctly reference this scholarly work, feel free to copy and paste the following:

Lin Wang and Zhixin Liu (2011). Robust Consensus of Multi-agent Systems with Bounded Disturbances, MultiAgent Systems - Modeling, Control, Programming, Simulations and Applications, Dr. Faisal Alkhateeb (Ed.), ISBN: 978-953-307-174-9, InTech, Available from: http://www.intechopen.com/books/multi-agent-systemsmodeling-control-programming-simulations-and-applications/robust-consensus-of-multi-agent-systems-withbounded-disturbances

\section{INTECH}

open science | open minds

\section{InTech Europe}

University Campus STeP Ri

Slavka Krautzeka 83/A

51000 Rijeka, Croatia

Phone: +385 (51) 770447

Fax: +385 (51) 686166

www.intechopen.com

\section{InTech China}

Unit 405, Office Block, Hotel Equatorial Shanghai

No.65, Yan An Road (West), Shanghai, 200040, China

中国上海市延安西路65号上海国际贵都大饭店办公楼 405 单元

Phone: +86-21-62489820

Fax: $+86-21-62489821$ 
(C) 2011 The Author(s). Licensee IntechOpen. This chapter is distributed under the terms of the Creative Commons Attribution-NonCommercialShareAlike-3.0 License, which permits use, distribution and reproduction for non-commercial purposes, provided the original is properly cited and derivative works building on this content are distributed under the same license. 